\title{
«Notre objectif: une formation plus orientée sur la pratique»
}

La nouvelle Ordonnance sur la formation professionnelle initiale et le plan de formation sont entrés en vigueur au début de cette année; l'année scolaire qui commencera en août $\mathbf{2 0 1 0}$ verra l'application des nouveautés, également dans la formation des assistantes médicales. Peter Tschudi, spécialiste en médecine générale à Urdorf/ $\mathrm{ZH}$ et président de la Commission de réforme concernant la formation des assistantes médicales, nous explique ce qui a changé.

Interview: Bruno Kesseli

Correspondance:

Dr Peter Tschudi

Médecine générale FMH

Feldstrasse $24 \mathrm{a}$

CH-8902 Urdorf

Tél. 0447345550

Fax 0447341821

peter.tschudi@greenmail.ch
Peter Tschudi, pourquoi était-il nécessaire de réformer la formation des assistantes médicales?

Peter Tschudi: L'Ordonnance sur la formation professionnelle a pour fondement la nouvelle loi fédérale sur la formation professionnelle (LFPrI) en vigueur

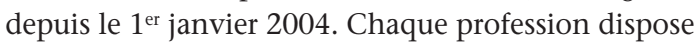
maintenant d'une ordonnance sur la formation, les employés de commerce comme les électroplastes, et justement aussi les assistantes médicales. La nouvelle ordonnance sur la formation professionnelle définit les compétences dont doivent disposer les apprentis pour exercer leur profession. Elle est entrée en vigueur au début 2010 après une phase de mise en œuvre qui a duré cinq ans et durant laquelle l'ancien règlement professionnel a été mis à jour et adapté aux conditions actuelles.

Quels étaient les membres de la Commission de réforme que vous avez présidée?

Outre la FMH, elle comprenait le Schweizerischen Verband Medizinischer PraxisAssistentinnen SVA et la Fédération suisse des associations d'assistantes médicales FSAAM, qui sont les deux associations de défense des intérêts des assistantes médicales. Des délégués des directions d'école, de l'Office fédéral de la santé publique, de la Confédération et des cantons y ont également participé.

www.mpaschweiz.ch - Informations complémentaires

A la rentrée scolaire 2010, la nouvelle Ordonnance sur la formation professionnelle entrera aussi en vigueur pour la formation des assistantes médicales. Vous trouverez des informations (en allemand) sur ce qui est resté et ce qui a changé sur www.mpaschweiz.ch. $Y$ figure entre autres le plan de formation pour les écoles professionnelles, les cours interentreprises et les entreprises. (Pour le texte en français, cliquer sur BiVo / Bildungsplan 04) MPA Bildungsplan $2010 \mathrm{fr}$ )

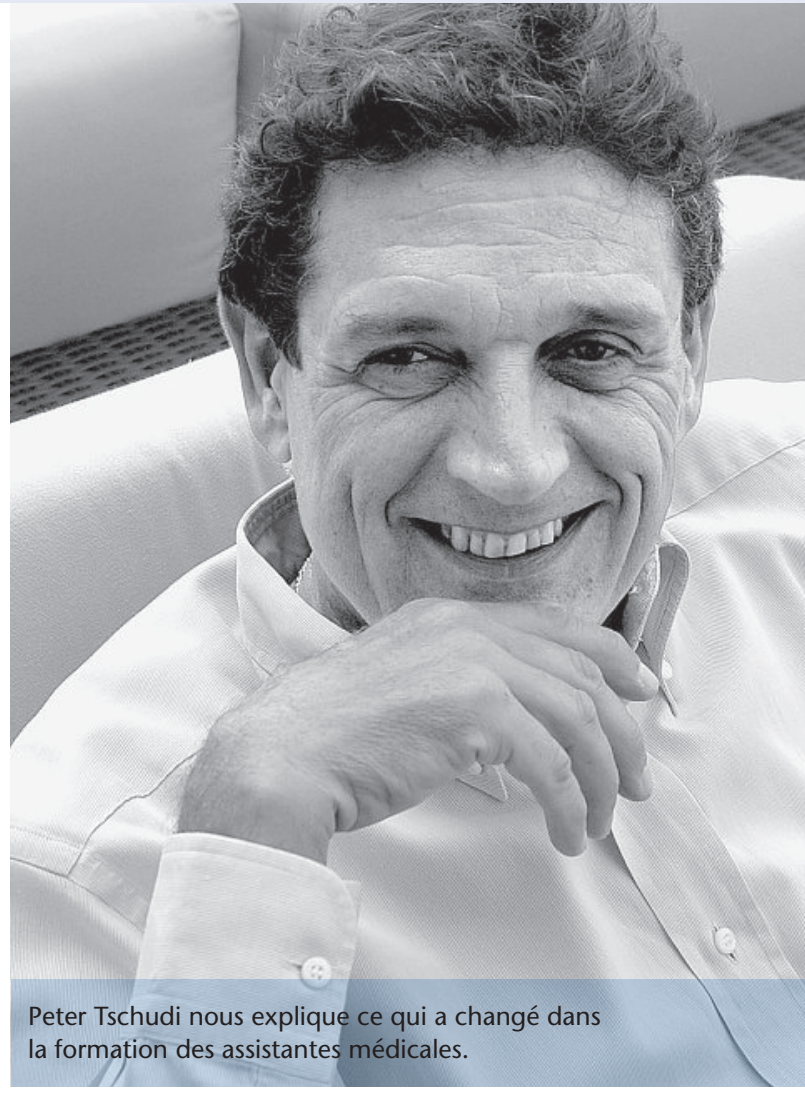

Les contenus concrets de la formation sont formulés dans le plan de formation. Tout a-t-il changé pour les assistantes médicales?

Aucune crainte; nous n'avons certainement pas voulu réinventer la roue. Un objectif important de la réforme était de rapprocher encore un peu plus la formation de la pratique et nous avons estimé que nous y parviendrions au moyen d'une démarche déductive, par exemple en ne commençant plus forcément par la cellule mais par des cas cliniques. Quels symptômes sont caractéristiques pour une infection pulmonaire? Comment puis-je reconnaître une thrombose veineuse profonde de la jambe? Ces questions sont prioritaires dans le travail quotidien des assistantes 
médicales. Certes, il est aussi intéressant et passionnant de connaître en détail la cascade de coagulation qui a lieu au niveau moléculaire et microscopique et qui conduit à boucher le vaisseau lésé, mais plutôt au sens d'un approfondissement. Le plan de formation va dans cette direction, en ce qui concerne les priorités.

\section{Quelles compétences va-t-on maintenant renforcer chez les} assistantes médicales?

L'accent principal sera mis sur la communication et les contacts avec les patients: comment aller à leur rencontre et les aborder, et comment adapter son discours en fonction de la situation? Les assistantes médicales pourront également parler de leurs expériences avec une personne spécialisée dans le cadre de groupes Balint* que l'on va encore créer. personnes aptes à diriger les groupes Balint (psychiatres ou médecins de premier recours expérimentés). Les personnes intéressées sont priées de prendre contact avec leur société cantonale de médecine ou le Dr Peter Tschudi par courriel: peter. tschudi@greenmail.ch

** $\quad$ www.fmh.ch $\rightarrow$ Services $\rightarrow$ Assistantes médicales $\rightarrow$ Documents à imprimer $\rightarrow$ Sélection du personnel dans le cabinet médical

\section{L'apport de nouveaux éléments doit généralement être compensé par la suppression d'anciens éléments pour conserver l'équilibre.}

Nous avons cherché concrètement à réduire les disciplines fondamentales des sciences naturelles telles que la physique et la chimie. On ne peut pas tout avoir et ces disciplines sont d'importance secondaire pour le travail des assistantes médicales. Celles-ci ne peuvent toutefois pas exercer leur activité sans connaissances

\section{Assistante médicale - un bref historique}

En 1971, la Fédération des médecins suisses a adopté le premier règlement sur la formation $d^{\prime}$ «aide médicale DVSA». Les personnes qui voulaient devenir assistante médicale devaient choisir entre un apprentissage de trois ans ou une formation de 2 ans $1 / 2$ dans une école privée.

Jusqu'au milieu des années 90 , la formation d'assistante médicale était réglée exclusivement par la FMH. Le premier règlement de l'OFIAMT est entré en vigueur au début 1996 en posant les fondements pour une formation reconnue sur le plan fédéral. A la même époque, la désignation a changé et l'aide médicale s'appelle désormais assistante médicale.

En 1999, la première volée d'apprenties a achevé avec succès la formation d'assistante médicale.

En 2010, la première réforme de la formation fédérale est appliquée en collaboration avec la FMH, les associations d'assistantes médicales et d'autres organisations.

\section{Informations utiles pour les médecins}

La FMH s'engage non seulement pour une formation convaincante des futures assistantes médicales mais elle offre aussi aux médecins avec des assistantes médicales, des documents utiles pour la gestion du personnel, l'évaluation du poste de travail et les salaires. En cliquant sur www.fmh.ch $\rightarrow$ SERVICES $\rightarrow$ Assistantes médicales, vous trouverez un grand nombre d'informations utiles concernant la sélection du personnel, les entretiens de collaborateurs, les cours de maîtres d'apprentissage et les recommandations salariales ainsi que des contrats de travail types.

de base en physique et en chimie. Pour les analyses de laboratoire et la radiographie notamment, activités «classiques» des assistantes médicales, il est important de posséder des connaissances de base en sciences naturelles, par exemple sur les radiations ou les réactions acide-base. Ces points continueront à faire l'objet d'un enseignement approprié.

Le nombre total d'heures a toutefois légèrement augmenté. Oui, principalement en raison des heures de sport qui sont passées de 100 à 200 heures suite à une directive de l'Office fédéral de la formation professionnelle et de la technologie (OFFT). Malgré tout, la plupart des cantons ont réussi à maintenir le nombre de jours d'école par semaine au niveau actuel.

A votre avis, y a-t-il d'autres nouveautés à mentionner? Il faut aussi souligner, me semble-t-il, que les examens radiologiques ne sont plus une discipline éliminatoire. Une note insuffisante à l'examen final peut donc être compensée et ne signifie plus obligatoirement un échec. En outre, la limite d'âge de 16 ans a été supprimée, conformément à une directive de l'OFFT, ce qui permet de commencer la formation d'assistantes médicales à la sortie de l'école obligatoire. La maitrise de la langue maternelle demeure une priorité élevée. En revanche, la langue étrangère ne doit plus être une langue nationale. Les écoles ont ainsi la liberté de proposer l'anglais comme langue étrangère et quelques cantons ont déjà prévu cette option.

Pourquoi les candidates à la formation d'assistante médicale n'ont-elles plus besoin de passer un examen d'admission?

Nous recommandons aux médecins de faire tout le nécessaire pour évaluer les candidates au préalable, d'autant plus que la limite d'âge de 16 ans n'est plus en vigueur. Ils devraient, si possible, prendre contact avec les enseignants des écoles secondaires et inviter les élèves intéressées à faire un stage de préapprentissage dans leur cabinet, et pas seulement pour une journée. C'est plus productif qu'un examen. Suite à des réflexions semblables, nous avons renoncé à demander aux candidates de fournir un certificat médical. La FMH offre différents moyens auxiliaires pour évaluer les aptitudes des candidates, moyens que l'on peut télécharger sur l'internet.**

\section{Une brève conclusion pour terminer?}

En termes de contenu, cette réforme produit des améliorations notables. Sur le plan organisationnel, les changements restent moindres, d'autant plus que les cantons les mettront en œuvre de façon différenciée. Mais ce n'est pas nouveau pour la Suisse. 